\title{
MARCHÉ LINGUISTIQUE
}

\section{Cyril Trimaille, Samuel Vernet}

Éditions de la Maison des sciences de l'homme | «Langage et société »

2021/HS1 Hors série | pages 229 à 232

ISSN 0181-4095

ISBN 9782735128273

DOI $10.3917 /$ ls.hs01.0230

Article disponible en ligne à l'adresse :

https://www.cairn.info/revue-langage-et-societe-2021-HS1-page-229.htm

Distribution électronique Cairn.info pour Éditions de la Maison des sciences de l'homme.

(c) Éditions de la Maison des sciences de l'homme. Tous droits réservés pour tous pays.

La reproduction ou représentation de cet article, notamment par photocopie, n'est autorisée que dans les limites des conditions générales d'utilisation du site ou, le cas échéant, des conditions générales de la licence souscrite par votre établissement. Toute autre reproduction ou représentation, en tout ou partie, sous quelque forme et de quelque manière que ce soit, est interdite sauf accord préalable et écrit de l'éditeur, en dehors des cas prévus par la législation en vigueur en France. Il est précisé que son stockage dans une base de données est également interdit. 


\section{Marché linguistique}

\section{Cyril Trimaille}

Université Grenoble Alpes

cyril.trimailleduniv-grenoble-alpes.fr

\section{Samuel Vernet}

Aix Marseille Université

samuel.vernet1dgmail.com

La notion de marché linguistique (désormais ML) renvoie au fait que, dans un espace socio-politique présentant une certaine unité, et dans toute situation d'interaction, les productions et variétés langagières se voient attribuer des valeurs différentielles et distinctives.

Le ML est un lieu de formation des prix des biens linguistiques, et la position relative des usages dans la hiérarchie des formes et des lectes est fonction de leur degré de conformité ou de proximité avec un ensemble de formes ou langues considérées comme standard, légitimes, dans l'absolu et dans une situation donnée. Il en résulte pour ces (ensembles de) formes linguistiques, un certain prestige, et pour les acteurs qui les détiennent une distinction, une autorité qui leur donnent accès à des ressources symboliques ou matérielles. Toute la structure sociale étant présente dans l'interaction (Bourdieu, 1977, p. 24), «il y a marché linguistique chaque fois qu'il y a interaction linguistique " (Encrevé, 1982, p. 100). Le ML doit donc être appréhendé à un niveau macro social, mais aussi au niveau micro de chaque interaction qui en est une 
actualisation. Toute production linguistique est le produit de la mise en relation d'un habitus linguistique et d'un ML, c'est-à-dire de l'ensemble des dispositions à s'exprimer (et percevoir) socialement acquises, mises en relations avec les lieux où se forment les prix des productions. Pour être écouté, cru, tout locuteur doit adapter sa production, en fonction de ce qu'il sait des valeurs des usages sur ces marchés. Dans un échange déséquilibré, le locuteur dominant peut imposer ses pratiques, sa langue, tandis que le locuteur dominé doit, pour obtenir un profit symbolique, s'aligner sur un certain modèle dominant - au risque de l'auto-censure, de l'hypercorrection, du silence.

La notion, forgée dans une série de textes par Pierre Bourdieu (principalement 1975 avec Boltanski, 1977, 1982), reprise et discutée par des linguistes proches (Encrevé, Laks, De Fornel), s'inscrit d'abord dans un appareil théorique sur l'économie des biens symboliques, aux côtés d'un ensemble de notions filant la métaphore économique (capital symbolique et linguistique, formation des prix, anticipation des coûts et profits). Elle participe ensuite à la critique de "l'autonomisation de la langue par rapport à ses conditions sociales de production " (Bourdieu, 1975 , p. 14) au centre des idéalisations saussurienne et chomskyenne de la langue.

Pour rependre un terme introduit dans la sociolinguistique anglophone des années 1970, on peut considérer que la langue légitime, dont la valeur sociale est la plus élevée sur le ML unifié, est la variété qui jouit d'un " prestige manifeste " selon l'expression de Peter Trudgill, tandis que certains habitus linguistiques oraux qui s'en éloignent ne peuvent prétendre qu’à un prestige latent sur un "marché franc ", sur lequel la règle de formation des prix differe de celles en vigueur sur le « marché unifié ». Mais pour Bourdieu, même les acteurs sociaux qui détiennent ces formes de franc-parler n'échappent pas, ou pas toujours, aux règles du ML unifié.

La notion de " compétence élargie ", définie par Bourdieu comme la capacité à maitriser les formes et leur caractère stylistiquement approprié pour les placer de façon idoine sur un ML, est très liée à celle d'habitus. Bien que Bourdieu ne cite nulle part Dell Hymes, cette notion est à rapprocher de celle de compétence de communication, comme le souligne la formulation suivante: " [L]a compétence chomskyenne est une abstraction qui n'inclut pas la compétence permettant d'utiliser adéquatement la compétence (quand faut-il parler, se taire, parler ce langage ou celui-là, etc.) »(Bourdieu, 1977, p. 18). 
S'il l'a illustrée par un certain nombre d'exemples, notamment dans Ce que parler veut dire, Bourdieu n’a jamais opérationnalisé sa théorie empiriquement. David Sankoff et Suzanne Laberge (1978), dans une exploitation du corpus de français parlé de Montréal, ont mis au point " une échelle d'intégration dans le marché linguistique ». L'indice d'intégration est conçu comme une variable sociale (parmi les autres variables indépendantes de la sociolinguistique variationniste : âge, emploi, classe sociale, sexe, ethnie, etc.), et elle est définie par ce critère : " the relative importance of the legitimized language in the socioeconomic life of the speaker» (1978, p. 241). Pour déterminer ce critère, huit sociolinguistes au fait de la situation sociolinguistique montréalaise classent 120 locuteurs sur l'échelle de participation au ML, et un indice allant de 0 (participation minimale au marché) à 1 (participation maximale) est attribué à chaque locuteur. L'accord entre les juges est massif, et l'indice créé semble mieux expliquer la présence de certaines variantes que d'autres facteurs sociologiques. William Kemp, Henrietta Cedergren et Jean Clermont, autres sociolinguistes québécois, ont opérationnalisé le degré d'insertion au ML. Cependant, pour Pierre Encrevé cette utilisation de la notion de ML réduite à une mesure de l'intégration au ML dominant ne tient pas compte de la multiplicité des marchés et de leur autonomie relative (Encrevé, 1982, p. 101).

Bernard Laks, dans son enquête sur les pratiques langagières de jeunes banlieusards (1983), propose une utilisation de la notion sans doute plus conforme à cette complexité. Il caractérise son terrain d'enquête (un centre de loisirs de Villejuif) comme un "marché où les pratiques linguistiques et sociales des agents sont évaluées [et dont il convient d'évaluer] l'effet propre de marché induit par le lieu où le groupe se réunit ». Il distingue ce marché de «l'espace social circonscrit par le groupe, [...] un marché symbolique sur lequel les membres, munis de leurs capitaux propres, entrent en lutte pour acquérir tel bien symbolique ou matériel » (Laks, 1983, p. 79).

Dans les années 1990, la Critical Discourse Analysis, entend prolonger les préoccupations bourdieusiennes sur le langage et le pouvoir (Kelly-Holmes, 2016, p. 162). Elle produit une analyse critique d'aspects discursifs de la globalisation, entendue comme l'unification de différents marchés (financiers, de biens et services, culturels), comme le sera, quelques années plus tard, la sociolinguistique critique. Dans ces approches, la notion de ML côtoie le marché économique et le marché du travail. Elle y est parfois entendue dans un sens très littéral de lieux (symboliques) où s'échangent concrètement les pratiques linguistiques 
transformées en produits - c'est-à-dire finalement, la façon dont les langues et les pratiques linguistiques sont marchandisées. La question des valeurs différentielles accordées aux pratiques linguistiques et des effets de domination sociale qui en découlent est toujours au cœur de la réflexion, mais la focale semble être placée plus précisément sur la/les langues comme ressources dans le capitalisme moderne.

$\mathrm{Si}$, combinée avec celle de langue légitime, la notion de ML semble aujourd'hui faire partie, en sociolinguistique, d'une sorte de fond commun pour désigner la hiérarchisation des ressources linguistiques, son appropriation l'aura en grande partie simplifiée et extraite de l'appareil théorique dans lequel elle prenait tout son sens, en articulation avec les notions bourdieusiennes de champs, d'habitus, de distinction, de reproduction.

\section{Références bibliographiques}

Bourdieu P. (1977), "L'économie des échanges linguistiques », Langue française 34, p. 17-34.

Bourdieu P. (1982), Ce que parler veut dire, Paris, Fayard.

Bourdieu P. \& Boltanski L. (1975), «Le fétichisme de la langue ", Actes de la recherche en sciences sociales 1 (4), p. 2-32.

Encrevé P. (1982), "À propos du "marché linguistique” ", dans Dittmar N. \& Schlieben-Lange B. (dir.), La sociolinguistique dans les pays de langues romanes, Tübingen, Gunther Narr Verlag, p. 97-103.

Laks B. (1983), "Langage et pratiques sociales ", Actes de la recherche en sciences sociales 46 , p. 73-97.

Sankoff D. \& Laberge S. (1978), « The linguistic market and the statistical explanation of Variability ", dans Sankoff D. (dir.), Linguistic Variation. Models and Methods, New York, Academic press, p. 239-250.

Renvois : Critique ; Langues régionales ; Marchandisation ; Pratique langagière. 